\title{
EFFECT OF MATURATION MEDIA SUPPLEMENTED WITH RETINOL OR ASCORBIC ACID OR $\alpha$-TOCOPHEROL ON CUMULUS CELL EXPANSION AND IN VITRO NUCLEAR MATURATION OF BUFFALO OOCYTES.
}

Khalil, W.A.; M.A. El-Harairy; A.E. Abdel-Khalek; and Z.M.R.K. Palani.

Animal Production Dept., Faculty of Agric., Mansoura Univ.

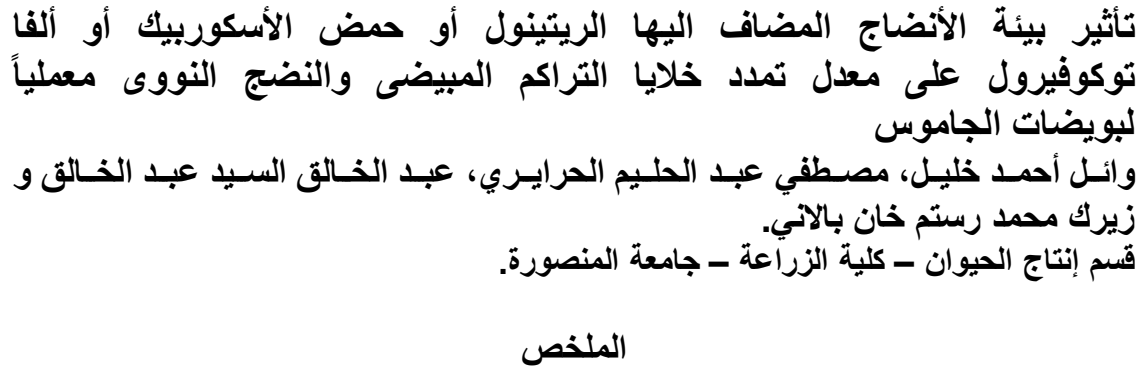

مقدمة

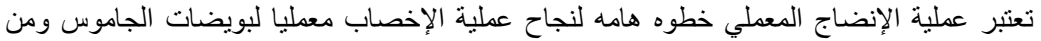

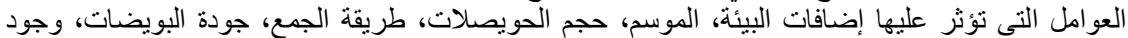

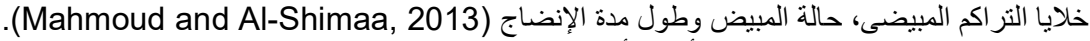

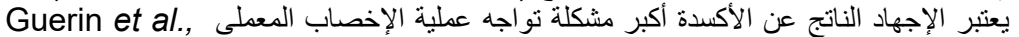
(2001) وقدعرف بعض الباحثين (Feugang et al., 2004) الإجهاد الناتج عن الإكسده على أنه ناتج

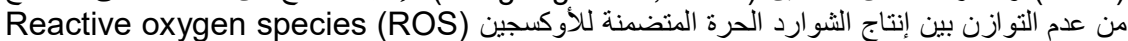

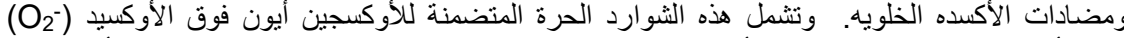

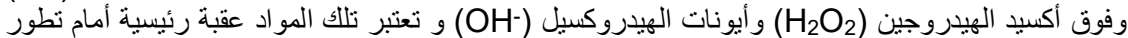
الأجنه (Guerin et al., 2001) حيث أنها تكون شديدة التفاعل مع جزيئات الخلايا المعقدة مثل الدهون والبروتينات والحمض النووي (DNA) وتسبب سلسله من تعطيل الوظائف مثل عدم تنشيط 
Khalil, W.A. et al.

Kowaltowski, 1999) وشذوذ الميتوكندريا (Halliwell and Gutteridge, 1989) الإنزيمات (and Vercesi

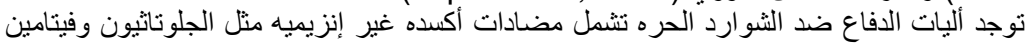

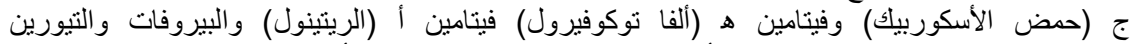

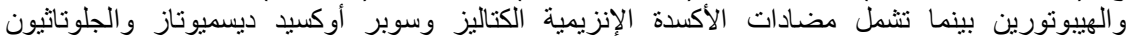

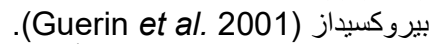

بعتبر فيتامين هـ مضاد أكسده طبيعى، حيث يوجد فيتامينى أه هـ هـ بكميات معنويه في المبيض أو

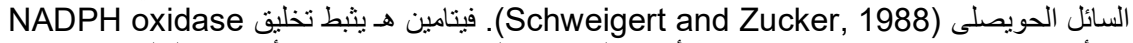

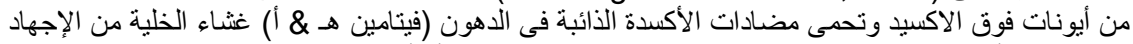

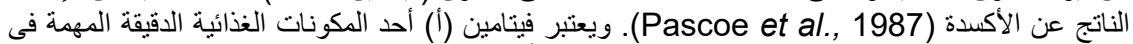

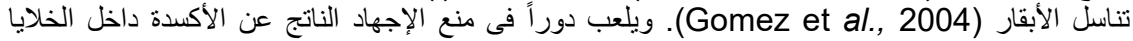
(Ahlemeyer et al., 2001)

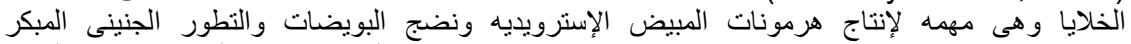

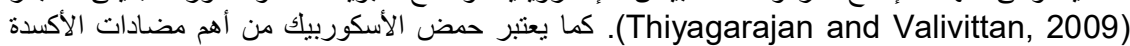

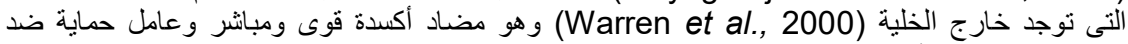

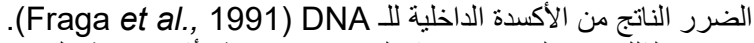

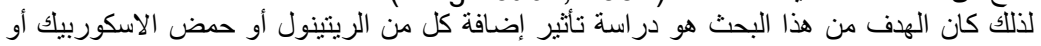

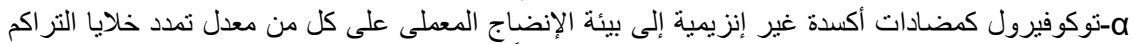

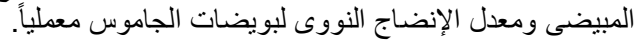

\section{الطرق والمواد}

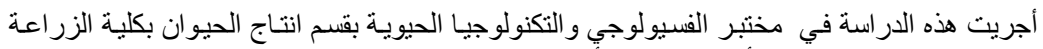

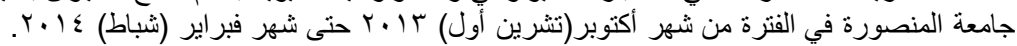

تم جمهع المبـايض من كل من المجـازر الاليه بمحافظـات القـاهرة و المنوفيـة (شبين الكوم) وكانت

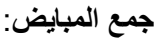

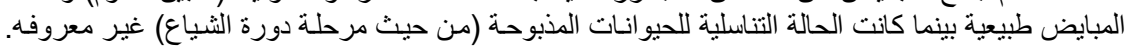

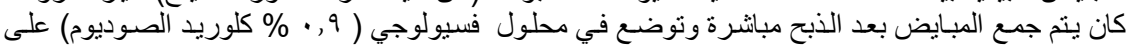

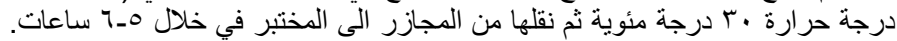
غسل المبايض:

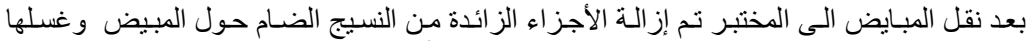

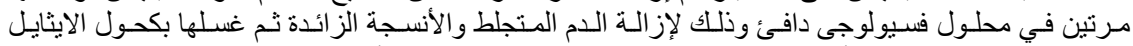

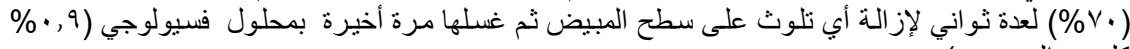
كلوريد الصوديوم). جمع البويضات:

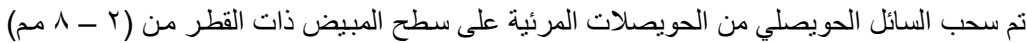

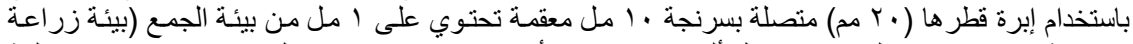

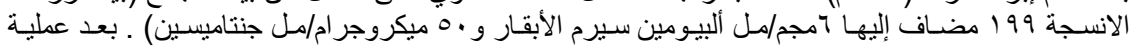

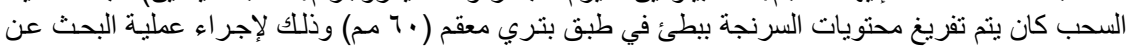
البويضات تحت الميكرسكوب الخاص بذلك وهو الإستريوميكرسكوب لتنقير العدد وتصنيف البويضات.

تقييم وتصنيف البويضات:

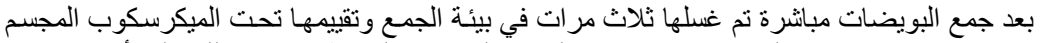

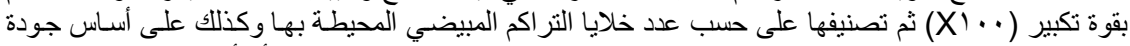

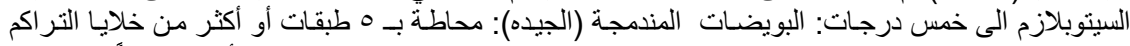

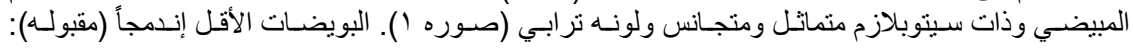




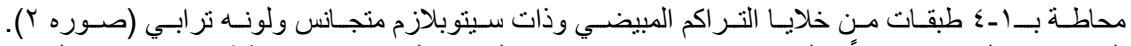

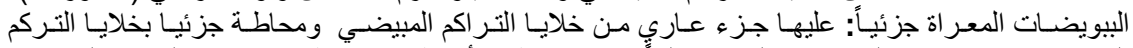

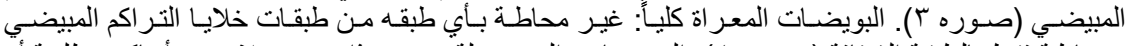

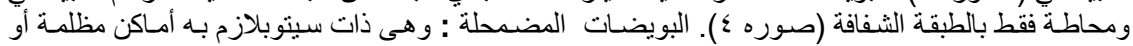

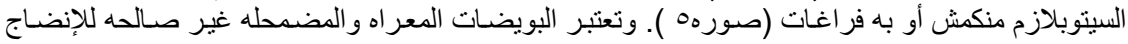

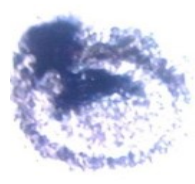

صوره (0): (0) بويضات مضمحلة

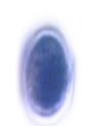

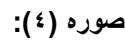
بويضات معراة كلياً

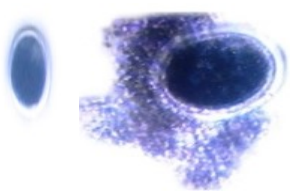

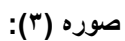
بويضات معراة جزئيا

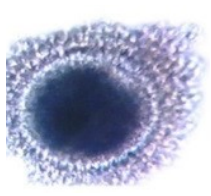

صورن (ب) إن: المعملى لذلك يتم إستبعادها.

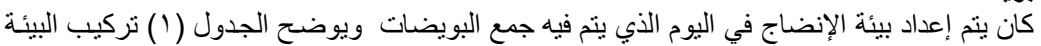

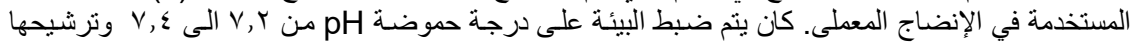
عن طريق فلتر قطره ب r , • ميكرومينر. بعد جمع وتقييم وتصنيف البويضات تم اختيار البويضات الجيدة والمقبولة (المندمجة والأقل إندماجاً)

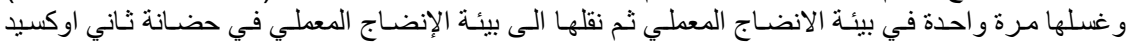

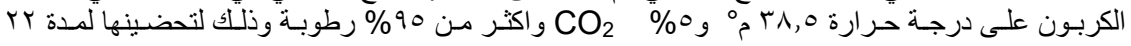

\begin{tabular}{|c|c|c|}
\hline \multicolumn{3}{|c|}{ جدول (1): تركيب بيئة الأنضاج المعملى } \\
\hline الشركة المنتجة & 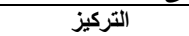 & محتويات البيئة \\
\hline Egyp. Orgn. Biol. Prod, Vacc. & 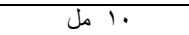 & البيئة زراعة الأنسجة_199 \\
\hline Sigma Chemi. Co. & 7مجم/مل & أل ألبيومين سيرم الآبقار \\
\hline Sigma Chemi. Co. & 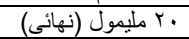 & صو صديوم بيروفات \\
\hline $\begin{array}{l}\text { Follitropin; Bioniche Animal Health, } \\
\text { Belleville, ON, Canada }\end{array}$ & م ميكرو جرام/ مل & FSH مون النطور الحويصلى \\
\hline $\begin{array}{l}\text { Egyptian Int. pharmaceutical Industries } \\
\text { Co. }\end{array}$ & • ا وحدة دولية/ مل & هرمون الأم الحامل HCG \\
\hline Sigma Chemi. Co. & | ميكرو جرام/ مل & هر مون الإستر اديول- V V \\
\hline Sigma Chemi. Co. & & جنتاميسين (مضاد حيوي) \\
\hline
\end{tabular}

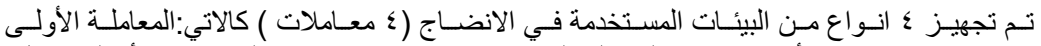

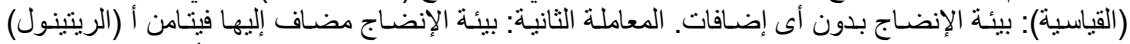

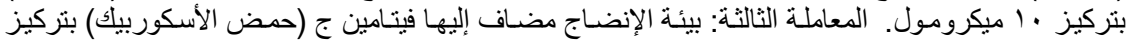

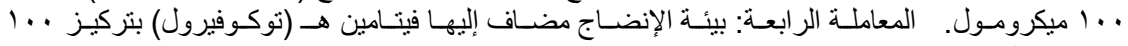

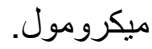
تصنيف البويضات بعد الانضاج حسب درجة تمدد خلايا التركم المبيضي (الأنضاج الستوبلازمى):

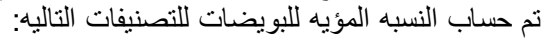

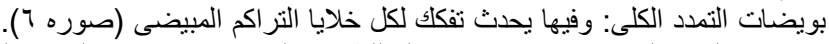

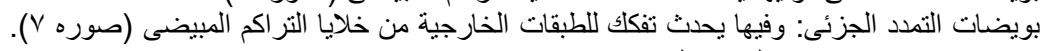

$$
\text { بويضات بدون تمدد خلايا التراكم المبيضي: (صوره م). }
$$




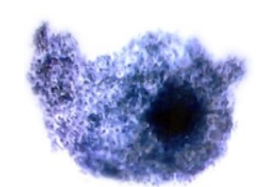

صوره رقم (V): يويضات التمدد الجزئى لخلايا

$$
\text { التراكم المبيضى لون }
$$

صوره رقم (T): بويضات التمدد الكلى لخلايا

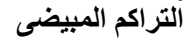

\section{صوره رقم (^): بويضات بدون تمدد لخلايا التراكم المبيضي}

تثييت البويضات وعملية الصبغ:

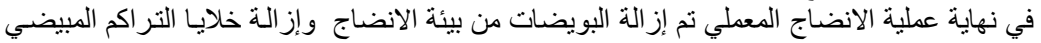

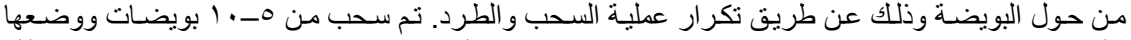

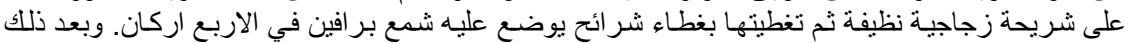

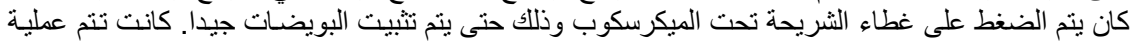

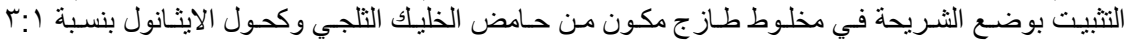

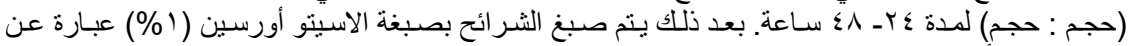

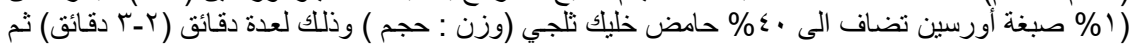

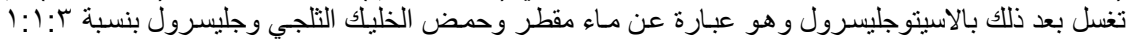

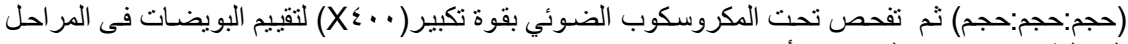

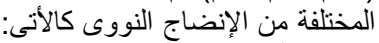

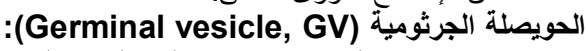
وفيها تكون الكروموسومات مغلفة المبرة بالغشاء النووي.

الحويصلة الجرثومية المتكسرة (Germinal vesicle breakdown, GVBD):

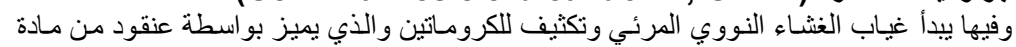
DNA بدون كروموسو مات فردية.

الطور الاستوائي الأول (Metaphase-I, M-I):

وفيها تتكاثف الكروموسومات في أزواج بدون ظهور الجسم القطبي الأول (صوره 9-1) (Aو). الطور الانفصالي الاول (Anaphase-I, A-I)

وفيها تبدأ الكرموسومات فى الإنفصال إلى مجموعتين مع ظهور خيوط المغزل (صوره • (1). الطور النهائي الاول (Telophase-I, T-I):

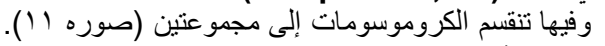
الطور الاستوائي الثاني (Metaphase-II, M-II):

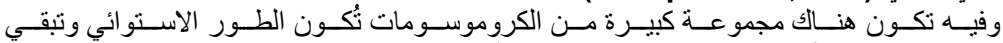

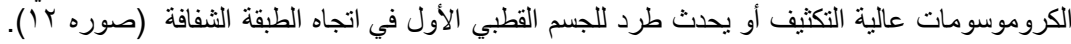

بويضات مضمحله (Degenerated): وفيها يكون الكروماتين متحوصل أو متبعثر أو عالي التكثيف. التحليل الأحصائى:

تم تحليل البيانات إحصائيا باستخدام برنامج (SAS 2004) باستخدام الحاسب الالي لإراسة التأثير

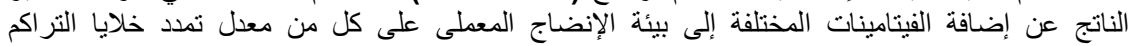
المبيضى والإنضاج النووي لبويضات الجاموس. تم مقارنة الفروق المعنوية باستخدام اختبار دانكن المنعدد 


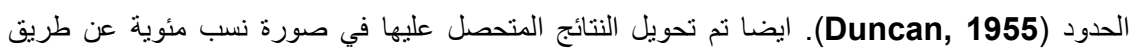
جداول الاركاسين ثم اعادتها مرة اخرى الى اصلها في الجداول المقدمة.
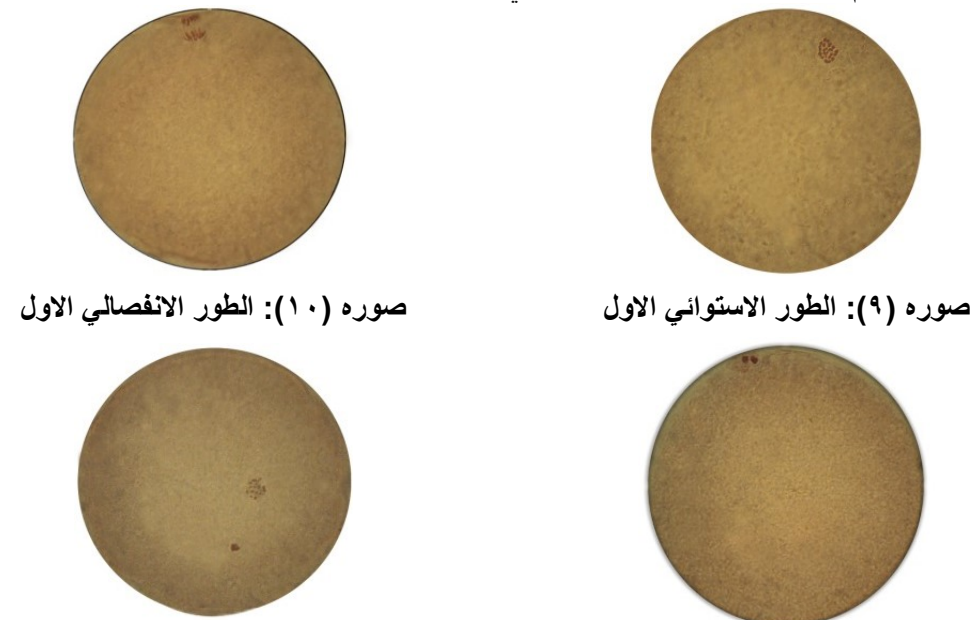

$$
\text { صوره (r r ): الطور الاستو ائي الثاني }
$$

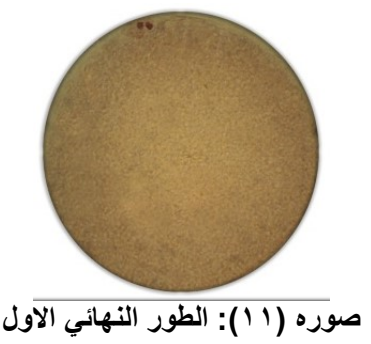

النتائج

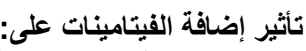

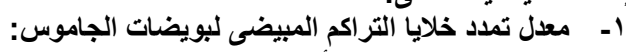

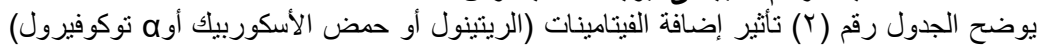

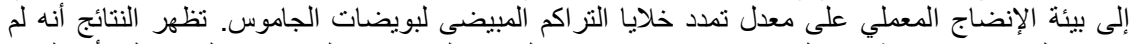

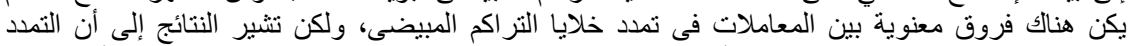

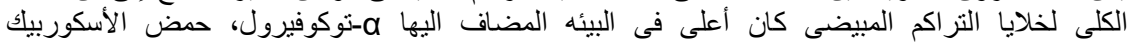

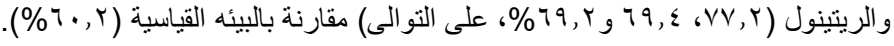


Khalil, W.A. et al.

جدول (ץ): تأثير إضافة الفيتامينات الى بيئه الأنضاج المعلى على معدل تمدد خلايا التراكم المبيضى

\begin{tabular}{|c|c|c|c|c|c|}
\hline \multirow{3}{*}{$\begin{array}{c}\text { الخطأ المعياري } \\
\text { (士) }\end{array}$} & & & & & لبويضات الجامر \\
\hline & \multicolumn{4}{|c|}{ بيئة الأتضاح المعطلى } & \multirow{2}{*}{ 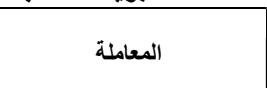 } \\
\hline & توكوفيرول & حض الأسكوربيك & الريتينول & القياسية & \\
\hline- & $\Lambda 4$ & 1.9 & 110 & 1.1 & عدد البويضات \\
\hline \multicolumn{6}{|r|}{ النسبه الموئه للبويضات (\%): } \\
\hline$\varepsilon, \Lambda$ & 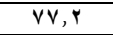 & $79, \xi$ & $79, r$ & $7 \cdot, Y$ & بويضات ذات تمدد كلى \\
\hline$\Gamma, \Gamma$ & 10,4 & $T V, r$ & 17,7 & YY,V & |بويضات تمدد جزئى \\
\hline$r, \mathrm{~V}$ & $v, \tau$ & $\overline{1 T, r}$ & $1 \varepsilon, \Gamma$ & $1 V, 1$ & بويضات غير متمددة \\
\hline$r, \mathrm{~V}$ & 94,0 & 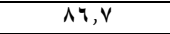 & $\Lambda \theta, V$ & $\Delta r, q$ & تمدد كلى + تمدد جزئي \\
\hline
\end{tabular}

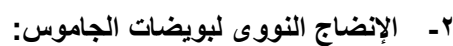

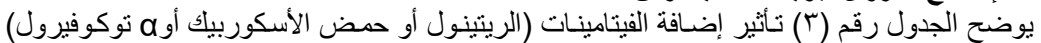

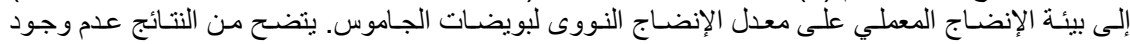

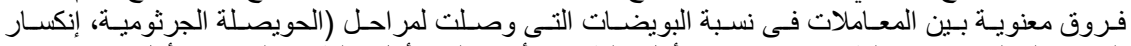

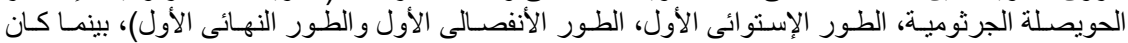

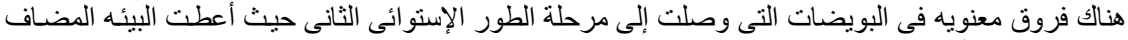

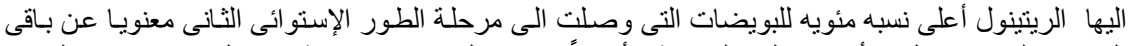

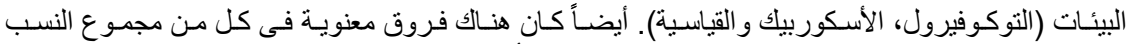

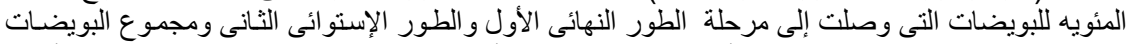

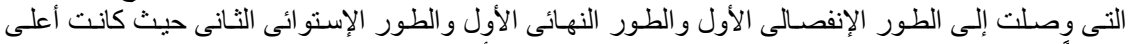
معنوياً فى البيئات المضاف اليها الريتينول، ه-توكوفيرول والأسكوربيك عن البيئه القياسية.

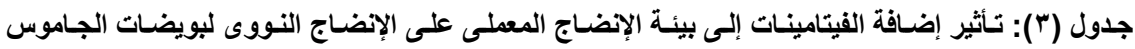

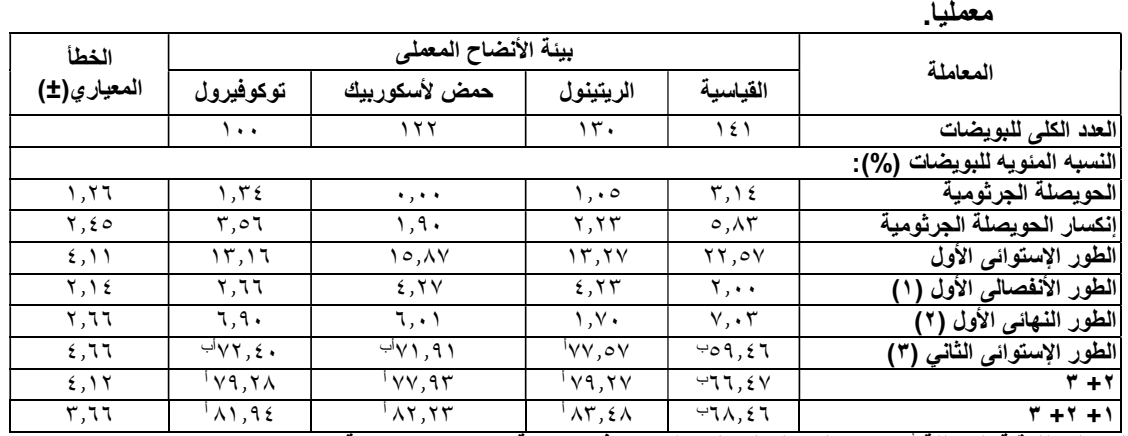

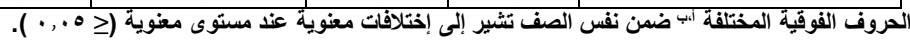

\section{المناقشة}

تم تقييم تأثثير إضافة الريتينول أو حمض الاسكوربيك أوه-توكوفيرول كمضادات أكسدة غير إنزيمية

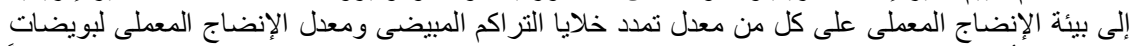

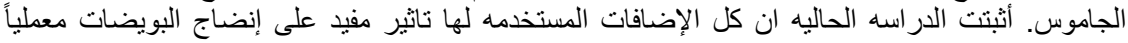

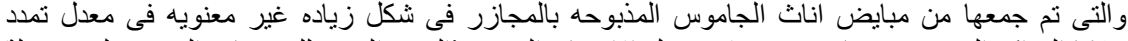

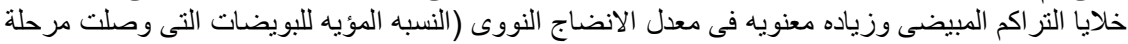

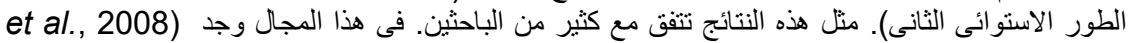
(Mlclea

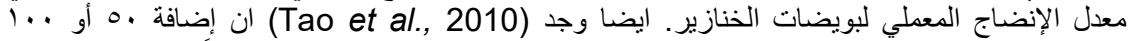

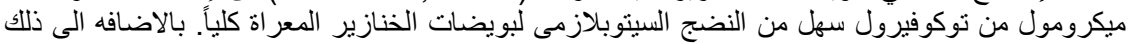
فإن (Kitagawa et al., 2004; Jeong et al., 2006) وجدوا أن إضافة فيتامين هـ بتركيز . . 1. 


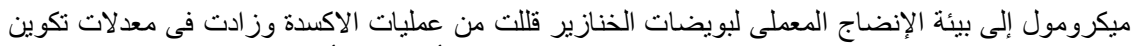

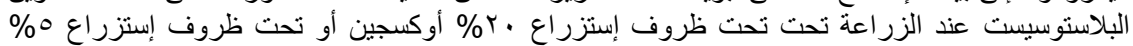

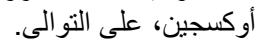

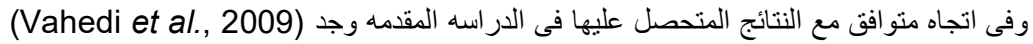

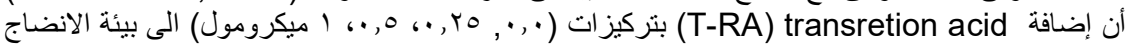

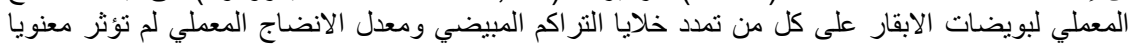

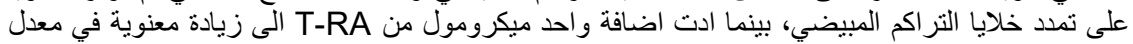

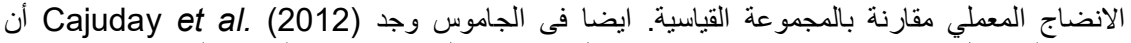

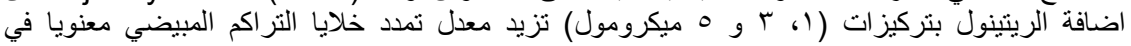

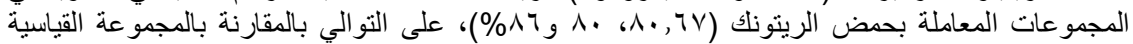

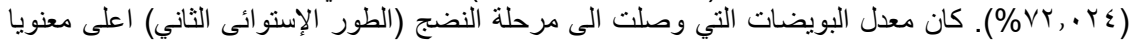

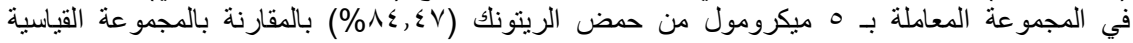
(\% \% TV, 9V)

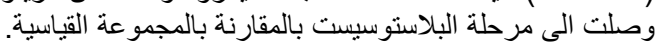

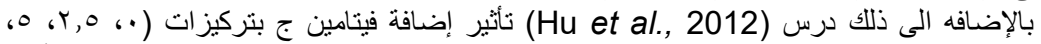

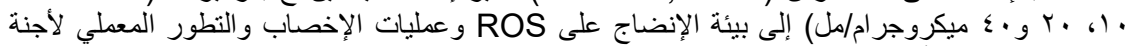

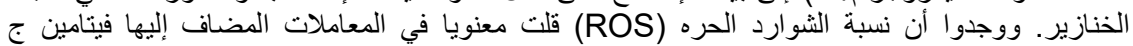

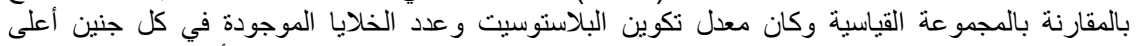

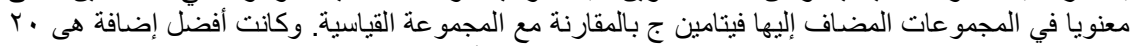

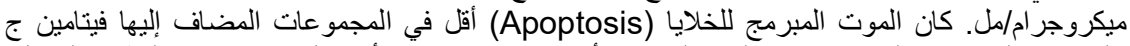

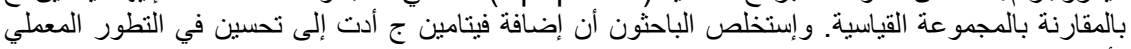

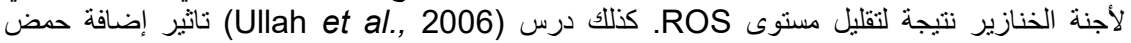

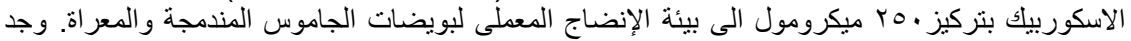

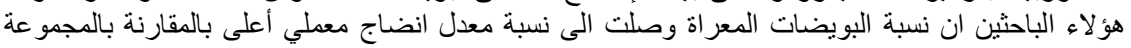

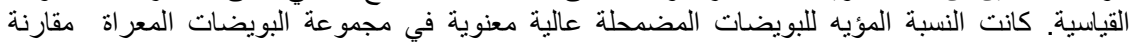

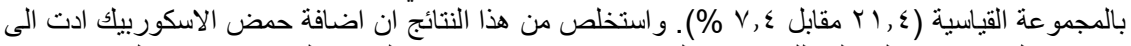

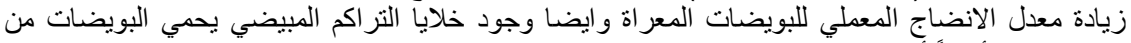

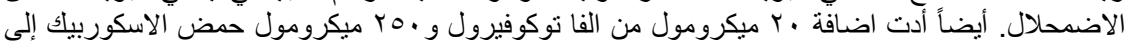

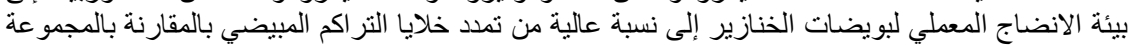

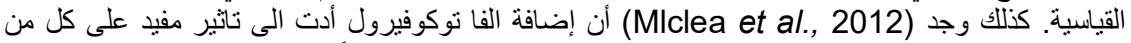
حيوية البويضة وتطور ها الى مرحلة الطور الإستو ائي الثناني وحسنت معنوياً من نطور نسبة الفية عالية من الاجنة

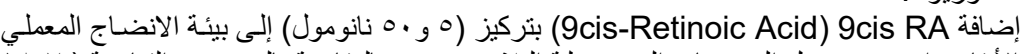

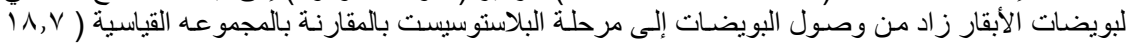

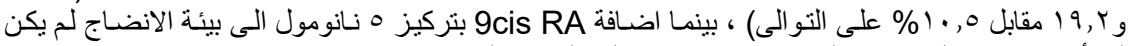

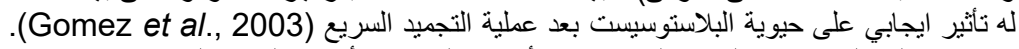

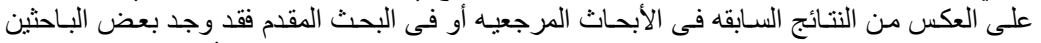

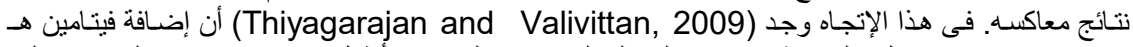

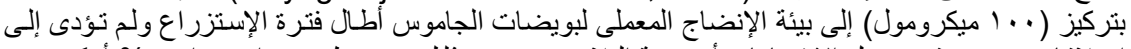

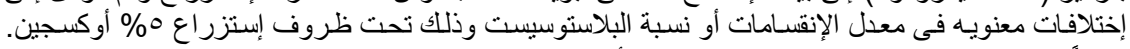

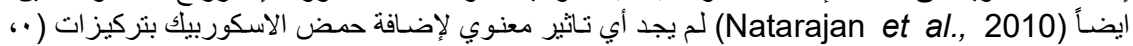

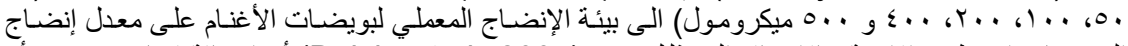

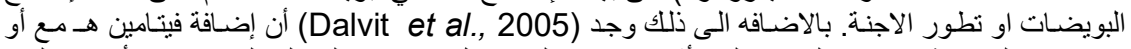

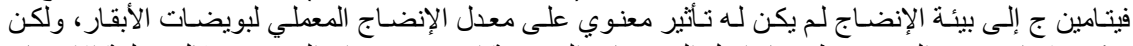

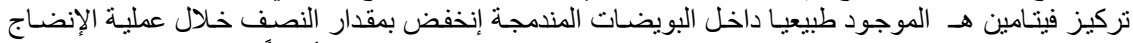

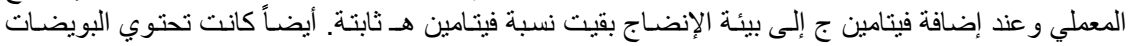

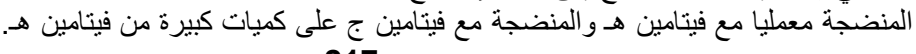


نستخلص من هذه النتائج أن إضافة كل من الريتينول أوحمض الأسكوربيك أو a-توكوفيرول

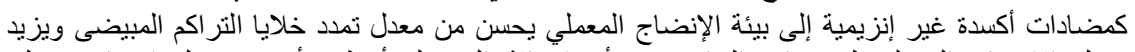

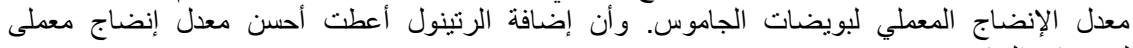

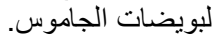

\section{REFERENCES}

Ahlemeyer, B; Bauerbach, E; Plath, M; Steuber, M; Heers, C; Tegtmeier, F. and Krieglstein, J. (2001). Retinoic acid reduces apoptosis and oxidative stress by preservation of SOD protein level. Free. Radic. Biol. Med., 30: 1067-1077.

Cajuday, L. A; Herrera, A. A. and Duran, D. H. (2012). Effect of retinoic acid on the development of Water buffalo embryos in vitro. Philipp. J. Vet. Anim. Sci., 38: 107-116.

Dalvit, G; Llanes, S. P; Descalzo, A; Insani, M; Beconi, M. and Cetica, P. (2005). Effect of alpha-tocopherol and ascorbic acid on bovine oocyte in vitro maturation. Reprod. Dom. Anim., 40: 93-97.

Duncan,D.B.(1955). Multiple range and multiple"F"test. Biometrics, 11: 1-42.

Feugang, J. M; Roover, R. D; Moens, A; Léonard, S; Dessy, F. and Donnay, I. (2004). Addition of $\beta$-mercaptoethanol or Trolox® at the morula/blastocyst stage improves the quality of bovine blastocysts and prevents induction of apoptosis and degeneration by prooxidant agents. Theriogenology, 61: 71-90.

Fraga, C.G; Motchnik, P.A; Shigenaga, M.K; Helbock, H.J; Jacob, R.A. and Ames, B.N. (1991). Ascorbic acid protects against endogenous oxidative DNA damage in human sperm. Proc. Natl. Acad. Sci. USA., 88:11003-11006.

Gomez, E; Roriguez, A; Goyache, F; Diez, C; Joseroyo, L; Moreira, P.N; Caamano, J.S; Moran, E. and Gutierrez-ada, A. (2004). Retinoid-dependent mRNA expression and poly-(A) contents in bovine oocytes meiotically arrested and/or matured in vitro. J. Reprod. Dev., 69: 101-108.

Gomez, E; Royo, L; Duque, P; Carneiro, G; Hidalgo, C;Goyache, F; Lorenzo, P. L; Alvarez, I; Facal. N. and Diez, C. (2003). 9-cis-Retinoic Acid During In Vitro Maturation Improves Development of the Bovine Oocyte and Increases Midkine but Not IGF-I Expression in Cumulus-Granulosa Cells.Molecular Reproduction and Development,66:247-255.

Guerin, P; El-Mouatassim, S; and Menezo, Y. (2001). Oxidative stress and protection against reactive oxygen species in the pre-implantation embryo and its surroundings. Hum. Reprod. Update, 7:175-189.

Halliwell, B. and Gutteridge, J. M. C. (1989). The chemistry of oxygen radicals and other derived species. In: Halliwell B, Gutteridge JMC, editors. Free radicals in biology and medicine. 2nd edition. Oxford: Clarendon Press; pp 22-85.

$\mathrm{Hu}$, J; Cheng, D; Gao, X; Bao, J; Ma, X. and Wang, H. (2012). Vitamin C enhances the in vitro development of porcine pre-implantation embryos by reducing oxidative stress. Reprod. Dom. Anim., 47: 873-879. 
Jeong, Y. W; Park, S. W; Hossein, M. S; Kim, S; Kim, J. H; Lee, S. H; Kang, S. K; Lee, B. C. and Hwang, W. S. (2006). Antiapoptotic and embryotrophic effects of a-tocopherol and L-ascorbic acid on porcine embryos derived from in vitro fertilization and somatic cell nuclear transfer. Theriogenology, 66:2104-2112.

Kitagawa,Y; Suzuki, K; Yoneda, A. and Watanabe, T. (2004). Effects of oxygen concentration and antioxidants on the in vitro developmental ability, production of reactive oxygen species (ROS), and DNA fragmentation in porcine embryos. Theriogenology, 62:1186-1197.

Kowaltowski, A. J. and Vercesi, A. E. (1999). Mitochondrial damage induced by conditions of oxidative stress. Free. Radic. Biol. Med., 26: 463-471.

Lopes, S; Jurisicova, A; Sun, J. G. and Casper, R. F. (1998). Reactive oxygen species: Potential cause for DNA fragmentation in human spermatozoa. Hum. Reprod., 13: 896-900.

Mahmoud, K.Gh.M. and Al-Shimaa Al-H.H. El-Naby (2013). Factors affecting buffalo oocytes maturation. Global Veterinaria, 11: 497-510.

Mlclea, I; Pacala, N; Zahan, M; Orlovshl, D; CÂRLEA, L. and Mlclea, V. (2012). Alpha-Tocopherol and ascorbic acid combinations have a positive effect on the maturation of pig oocytes. Bulletin UASVM Animal Science and Biotechnologies, 69: 269-276.

Mlclea, I; Zahan, M; Andrea, H; Mlclea, V. and Roman, I. (2008). The effect of several a-tocopherol concentrations on swine oocyte maturation. Bulletin UASVM Animal Science and Biotechnologies, 65: 366-370.

Natarajan, R; Bhawani, S.M; and Munuswamy, D. (2010). Effect of $\alpha$ tocopherol supplementation on in vitro maturation of sheep oocytes and in vitro development_of preimplantation sheep embryos to the blastocyst stage. J. Assist. Reprod. Genet.,27: 483-490.

Pascoe, G; Olafs dottier, F. and Read, D. (1987). Vitamin E protection against chemical induced cell injury: Maintenanceof cellular protein thiols as a cytoprotective mechanism. Arch. Biochem. Biophys., 256: 150-158.

SAS (2004). SAS/Stat. User's Guide Static's, Ver., 6.06 4th Ed. SAS Institute Inc. Cary, NC.

Schweigert, F.J. and Zucker, H. (1988). Concentrations of vitamin A, $\beta$ carotene and vitamin $E$ in individual bovine follicles of different quality. J. Reprod. Fert., 82: 575-579.

Tao, Y; Chen, H; Tian, N. N; Huo, D. T; Li, G; Zhang, Y. H; Liu, Y; Fang, F.G; Ding, J. P. and Zhang, X. R. (2010). Effects of L-ascorbic acid, $\alpha-$ tocopherol and co-culture on in vitro developmental potential of porcine cumulus cells free oocytes. Reprod. Dom. Anim., 45: 19-25.

Thiyagarajan, B. and Valivittan, K. (2009). Ameliorating effect of vitamin E on in vitro development of preimplantation buffalo embryos. J. Assist. Reprod. Genet., 26:217-225.

Ullah, L; Jalali, S; Khan, H; Shami, S. A. and Kiyani, M. M. (2006). Effect of Lascorbic Acid on Ravi Buffal Oocytes During in vitro Maturation. Pakistan Journal of Biological Sciences, 9: 2369-2394.

Vahedi, V; Zeinoaldini, S; Kohram, H. and Farahavar, A. (2009). Retinoic acid effects on nuclear maturation of bovine oocytes in vitro. Afr. J. Biotechnol., 8: 3974-3978.

Warren, S; Patel, S. and Kapron, C.M. (2000). The effect of vitamin E exposure on cadmium toxicity in mouse embryo cells in vitro. Toxicology, 142: 119-126. 


\section{EFFECT OF MATURATION MEDIA SUPPLEMENTED WITH RETINOL OR ASCORBIC ACID OR $\alpha$-TOCOPHEROL ON CUMULUS CELL EXPANSION AND IN VITRO NUCLEAR MATURATION OF BUFFALO OOCYTES. \\ Khalil, W.A.; M.A. El-Harairy; A.E. Abdel-Khalek; and Z.M.R.K. Al-Palani. Animal Production Dept., Faculty of Agric., Mansoura Univ.}

\section{ABSTRACT}

The main objective of this study was to evaluate the effect of supplementing maturation media with, retinol $(10 \mu \mathrm{mol} / \mathrm{ml})$, ascorbic acid $(100 \mu \mathrm{mol} / \mathrm{ml})$ and $\alpha$ tocopherol $(100 \mu \mathrm{mol} / \mathrm{ml})$, on cumulus cell expansion and in vitro nuclear maturation of buffalo oocytes. Oocytes were collected from ovaries taken from slaughterhouses. Only compact oocytes were used for in vitro maturation by TCM-199 medium supplemented with different vitamin levels for $22 \mathrm{~h}$ in $\mathrm{CO}_{2}$ incubator at $38.5{ }^{\circ} \mathrm{C}, 5 \%$ $\mathrm{CO}_{2}$ and $95 \%$ humidity. Maturation rate was determined in terms of degree of expansion of cumulus cells and oocytes at metaphase-II (nuclear maturation). Results revealed that all supplements yielded higher percentages of oocytes showing full expansion of cumulus cells $(77.2,69.4$ and $69.2 \%$ for tocopherol, ascorbic acid and retinol, respectively) as compared to control medium $(60.2 \%)$, but the differences were not significant. Maturation rate in term of percentage of oocytes arrested at anaphase-I, telophase-I and metaphase-II was significantly higher for media supplemented with retinol, tocopherol and ascorbic acid, being 83.5, 82.2 and $81.94 \%$ as compared to $68.54 \%$ in control medium, respectively.

Conclusively, the present results may suggest that the supplementation of maturation medium (TCM-199) with retinol, ascorbic acid and $\alpha$-tocopherol as a non enzymatic antioxidants had a beneficial effect on the cumulus cell expansion and nuclear maturation of buffalo oocytes. Retinol supplementation yielded the best results.

Keywords: Buffalo oocytes, in vitro maturation, non enzymatic antioxidants.

كلية الزراعة - جامعة المنصورة مركز البحوث الزراعية
قام بتحكيم البحث

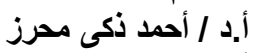

أ.د / علاء فؤاد الدين محرز 